\title{
Una aplicación de los sistemas de predicción por con- juntos para avisos meteorológicos
}

Los sistemas de predicción por conjuntos son herramientas de vital importancia para la emisión de avisos meteorológicos. Dada su naturaleza, pueden explorar el espacio de estados atmosféricos posibles ofreciendo un abanico de escenarios meteorológicos, algunos de los cuales pueden dar lugar a fenómenos adversos. Pero la cantidad de información que brindan los SPC es ingente, por lo que es de vital importancia condensarla con métodos de síntesis adecuados sin perder información relevante. En este capítulo presentamos una aplicación informática desarrollada en AEMET que, basándose en estos principios, ofrece a los predictores del tiempo una serie limitada de mapas que les permite tomar decisiones en tiempos reducidos.

Palabras clave: sistemas de predicción por conjuntos aplicados a la gestión de emisión de avisos meteorológicos, avisos meteorológicos, herramienta de predicción probabilista para avisos meteorológicos. 


\subsection{Introducción}

Los sistemas de predicción por conjuntos (SPC) proporcionan gran cantidad de información: para un sólo lugar y para cada uno de los parámetros meteorológicos de interés el sistema proporciona un conjunto de predicciones distintas, 51 en el caso del ECENS [3] (sec. 19.3 en la página 293) del ECMWF (cap. 19 en la página 289). Es interesante disponer de herramientas que analicen automáticamente las predicciones de cada miembro del SPC y reduzcan el volumen de información adecuadamente, para hacerlo más accesible al predictor o usuario final. Existen varias formas de reducir esta información y los métodos difieren en función del objetivo que se persiga. En el caso de la elaboración de un aviso meteorológico por un fenómeno adverso, importa conocer cuán extremos o normales son los valores predichos por el SPC. El Extreme Forecast Index (EFI, [2], sec. 27.7 en la página 419) compara la distribución predicha por el ECENS con la distribución de la climatología del propio ECENS. Este indicador es útil, ya que destaca las situaciones poco probables y, por tanto, potencialmente adversas.

En este capítulo se describe una aplicación relativamente sencilla. A diferencia de indicadores como el EFI, que hace uso de la climatología del SPC, se hace la suposición de que se dispone de un SPC calibrado, que puede usarse directamente en la predicción de fenómenos adversos, en particular viento, temperaturas extremas, lluvia y nieve. En el caso de estudio que incluimos al final del capítulo se usan las predicciones directas del ECENS, pero el mismo esquema puede aplicarse a cualquier otro tipo de SPC, calibrado mediante un postproceso o no. Se trata de resumir las predicciones de un SPC, orientando el resultado a proporcionar una ayuda en la elaboración de un aviso meteorológico. Para ello se tendrán en cuenta los umbrales que definen cada uno de los niveles de aviso (amarillo, naranja y rojo) en cada una de las zonas previamente definidas y para cada uno de los fenómenos considerados.

El capítulo comienza explicando someramente qué es un aviso meteorológico. En la siguiente sección se comenta qué parámetros se calculan en la aplicación a partir de las predicciones del ECENS, relacionados con la probabilidad de ocurrencia, la extensión del área afectada, el nivel máximo de aviso esperado y el más plausible. Finalmente, se ilustra la herramienta analizando brevemente un caso concreto, la borrasca ANA, que afectó principalmente a la mitad norte peninsular durante los días 10 y 11 de diciembre de 2017.

\subsection{Avisos meteorológicos}

Los avisos meteorológicos para el público en general se limitan a un número de supuestos. En el Plan Nacional de Predicción y Vigilancia de Fenómenos Meteorológicos Adversos (Meteoalerta [1], cap. 41 en la página 627) se contemplan: lluvias, nevadas, vientos, tormentas, temperaturas extremas, fenómenos costeros (viento y mar), polvo en suspensión, aludes, galernas cantábricas, risagas en Baleares, nieblas, deshielos, olas de calor y de frío y tormentas tropicales.

Los niveles de aviso se asocian a colores, lo que permite una fácil lectura. Los colores corresponden a distintos niveles de riesgo: el verde indica situaciones en las que no se prevé riesgo y el amarillo, naranja y rojo significan riesgo bajo, importante o extremo. Concretando algo más, el nivel amarillo no indica riesgo para la población general, pero sí para alguna actividad concreta. El naranja indica riesgo de fenómenos meteorológicos no habituales y con cierto grado de peligro para actividades usuales. El rojo indica riesgo de fenómenos de intensidad excepcional y con un riesgo para la población muy alto.

Los niveles de aviso se determinan mediante umbrales que en algunos casos dependen de la zona y en otros no. En la actual versión de Meteoalerta, los avisos con umbrales zonificados son los de temperaturas máximas y mínimas, lluvias, nevadas y vientos. Estos umbrales están publicados (por ejemplo en la página web de Agencia Estatal de Meteorología (AEMET), http://www.aemet. es/documentos/es/eltiempo/prediccion/avisos/plan_ meteoalerta/umbrales_niveles_aviso.pdf).

Los niveles de aviso se establecen tratando de armonizar dos criterios: en primer lugar el nivel de adversidad, en función del peligro que pueda derivarse de una determinada situación meteorológica y, en segundo lugar, la climatología, ya que las situaciones de aviso deben ser poco frecuentes desde el punto de vista del clima. Es decir, un aviso rojo debe ser, al mismo tiempo, adverso y poco frecuente. Por ejemplo, en el Prepirineo de Barcelona se dan nevadas frecuentemente, mientras que en Sevilla no. El nivel de peligro, para una misma cantidad de nieve acumulada, es mayor en Sevilla que en el Prepirineo de Barcelona, porque la 
actividad humana se adapta al clima. Por tanto, los niveles de aviso tienen un grado de arbitrariedad a tener en cuenta, ya que a veces no es fácil establecer ese equilibrio entre el peligro del aviso y la frecuencia de ocurrencia. Continuando con el ejemplo, los umbrales de aviso (amarillo, naranja y rojo) de nieve acumulada en 24 horas son 2, 5 y $20 \mathrm{~cm}$ para Sevilla y 5,20 y $40 \mathrm{~cm}$ para el Prepirineo de Barcelona. Por tanto, una nevada de $5 \mathrm{~cm}$ supone un aviso amarillo en el Prepirineo de Barcelona y naranja en Sevilla.

En los avisos que emite AEMET se señala el grado de probabilidad de ocurrencia del fenómeno adverso. Se hace mediante tres tramos de probabilidad de ocurrencia: por debajo del $10 \%$, entre $10 \%$ y $40 \%$, entre $40 \%$ y $70 \%$, y más del $70 \%$, que se pueden calificar como improbables, posibles, probables y muy probables, respectivamente. La probabilidad de ocurrencia de un fenómeno puede estimarse con un SPC, dividiendo el número de predicciones positivas entre el total de predicciones. Así, si en una predicción de un SPC de 51 miembros, como el ECENS en su configuración actual, hay 15 miembros que predicen la superación de un determinado umbral de aviso, se estima que habrá una probabilidad del $29 \%$ de que efectivamente así suceda. Esto, claro está, en el supuesto de que el ECENS sea un sistema de predicción bien construido y que todos sus miembros sean equiprobables (sec. 13.7.2 en la página 191).

Además de la probabilidad de ocurrencia, es interesante conocer la extensión del área afectada. El área afectada por un determinado fenómeno adverso es uno de los criterios que se tienen en cuenta a la hora de decidir emitir un aviso. En general, el aviso no se emite, o se reduce el nivel de adversidad, si el fenómeno va a afectar un área muy reducida. El área afectada puede estimarse directamente a partir de las predicciones del SPC, contabilizando el porcentaje de puntos de malla (ver sec. 10.6 en la página 141) que, dentro de una zona, superan el umbral de aviso.

Otro parámetro de interés es el nivel máximo aviso en cada área, es decir, el valor más adverso de todos los predichos por los miembros del SPC en una determinada zona y para un determinado fenómeno, independientemente de que sea más o menos probable o más o menos extenso. Para ilustrarlo, imaginemos que el SPC predice un nivel máximo naranja para temperaturas máximas en la provincia de Sevilla. En este caso sabremos que algún miembro del SPC predice, en algún punto de malla dentro de la provincia de Sevilla, una temperatura máxima superior al umbral naranja, no llegándose a superar el umbral rojo en ningún caso.

La aplicación que describimos en este capítulo se limita a avisos por lluvias (acumulación en 12 horas), nevadas (acumulación en 24 horas), vientos y temperaturas extremas (máximas y mínimas). Como se explica con más detalle en la siguiente sección, la aplicación se centra en resumir la enorme cantidad de información procedente de un SPC, orientando el esfuerzo hacia la estimación de la probabilidad de ocurrencia, la extensión del área afectada y el nivel máximo de aviso previsto.

\subsection{Un problema de probabilida- des}

Imaginemos un área problema: el Prepirineo de Barcelona; un tipo de aviso: aviso rojo de nieve acumulada en $24 \mathrm{~h}$, con un umbral de $40 \mathrm{~cm}$ para el área correspondiente; y un período de predicción: el día 12-03-2018, suponiendo que fuese mañana o pasado y nos interese si emitir o no aviso. Supongamos que disponemos de las predicciones procedentes de 51 miembros del ECENS, donde cada uno de estos miembros proporciona la acumulación de nieve prevista en una malla de posiciones geográficas uniformemente distribuidas, en este ejemplo dentro de la provincia de Barcelona, particularmente en el área del Prepirineo de Barcelona (PPB). Nos planteamos tres sencillos problemas de probabilidades.

En primer lugar, queremos saber la probabilidad de que, en algún lugar del área PPB se supere el umbral rojo. Es decir, no estamos interesados en la extensión del fenómeno, de si va a afectar a un área amplia o reducida dentro del PPB. Estamos interesados en saber la probabilidad de que se supere el umbral de $40 \mathrm{~cm}$ en algún lugar del PPB. Esta probabilidad se puede estimar directamente con la proporción de miembros del SPC que prevén acumulaciones por encima de $40 \mathrm{~cm}$, como mínimo en un punto de la malla. En este cálculo, se supone implícitamente que las predicciones dadas por cada uno de los miembros del SPC son igualmente plausibles, es decir, equiprobables. Llamamos a este valor probabilidad de superación del umbral (ver Figura 35.1 en la página siguiente columna izquierda).

Para disponer de una idea de la extensión del área afectada por el aviso planteamos este segundo problema: ¿cual es el área afectada esperada, suponiendo 
que se va a superar el umbral de aviso? La respuesta se calcula en dos pasos. En primer lugar se calcula la proporción de puntos de malla que superan el umbral de aviso, $40 \mathrm{~cm}$ en este caso, para cada uno de los miembros del SPC. La proporción de puntos de malla que superan el umbral es una estimación de la proporción de área afectada, porque los puntos de malla están distribuidos uniformemente. En segundo lugar, se calcula el promedio de estas proporciones, seleccionando los miembros del SPC que superan el umbral en algún punto. Llamamos a este valor proporción de área afectada, donde se supone implícitamente que sólo se tienen en cuenta aquellas predicciones, o miembros del SPC, que superan el umbral del aviso en algún punto (ver Figura 35.1 columna derecha).
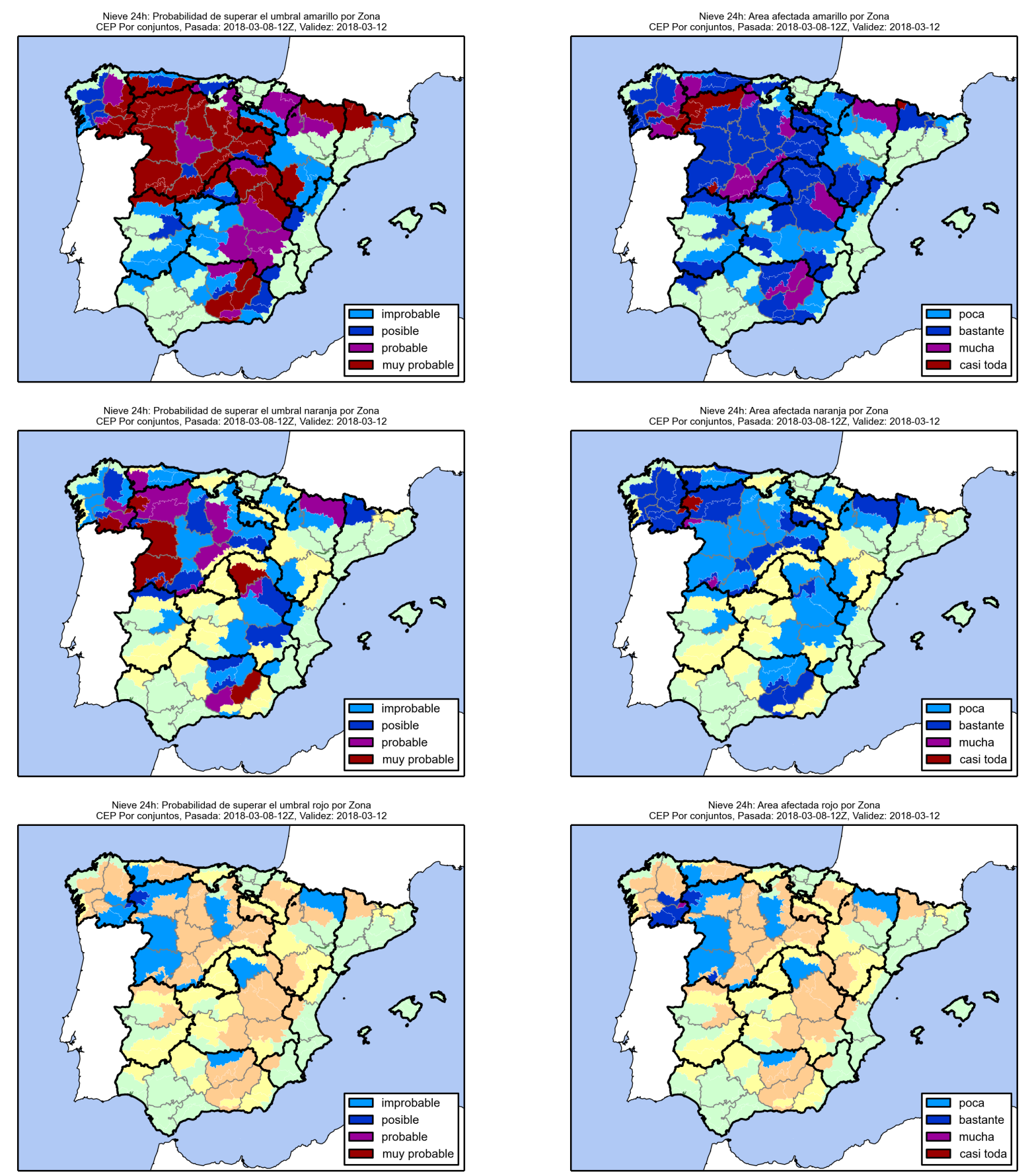

Figura 35.1: Avisos por nieve acumulada en 24 horas: probabilidad de superación del umbral (izquierda) y proporción de área afectada (derecha) para el día 12-03-2018. Las filas corresponden a los umbrales de amarillo, naranja y rojo. 
Con estos dos valores, probabilidad de superación del umbral y proporción de área afectada (Figura 35.1 en la página anterior), ya se dispone de una idea de lo que el SPC está prediciendo para un problema concreto, en este ejemplo, la superación del umbral rojo por acumulación de nieve en $24 \mathrm{~h}$ en el Prepirineo de Barcelona para el día de mañana, supuestamente el 12-03-2018. Al mismo tiempo y, esto es interesante para el uso práctico de un SPC, se reduce el volumen de información.

¿Cómo interpretar estos dos valores, la probabilidad de superación del umbral y la proporción de área afectada? Ponemos algunos ejemplos. Valores bajos de probabilidad y altos de área afectada nos indican que pocos miembros del SPC están superando el umbral, pero aquellos pocos que lo superan predicen áreas afectadas extensas (es decir, hay probabilidad baja de que se produzca un fenómeno adverso extenso). Por el contrario, valores altos de probabilidad y bajos de área afectada nos indican que muchos miembros del SPC superan el umbral, pero, en promedio, el área afectada predicha es pequeña (es decir, la probabilidad de que ocurra el fenómeno adverso es alta, pero no se espera que sea extenso). Hay casos en los que la interpretación es más sencilla: valores simultáneamente bajos de probabilidad y de área afectada indican que pocos miembros del SPC predicen superación del umbral y, además, el área afectada en los casos que se supere el umbral es pequeña. Por el contrario, la situación más adversa se dará con probabilidades y áreas afectadas altas: en este caso muchos miembros del SPC superan el umbral, y además coinciden en que el área afectada será extensa.

Finalmente, nos podemos preguntar: ¿cual es la probabilidad de que se supere el umbral de aviso en un punto cualquiera del área elegida? En nuestro ejemplo la pregunta sería: ¿con qué probabilidad se superará mañana el umbral de $40 \mathrm{~cm}$, situándonos en un lugar cualquiera del Prepirineo de Barcelona? La respuesta se puede dar en función de los dos parámetros ya explicados, puesto que es el producto de la probabilidad de superación del umbral y la proporción de área afectada. Por ejemplo: si la probabilidad de superación del umbral es del $50 \%$ y la proporción de área afectada del $30 \%$, la probabilidad de que se supere el umbral de aviso en un punto cualquiera del área considerada es el producto correspondiente $(0.5 \times 0.3=0.15)$, es decir, el $15 \%$.

En la aplicación que describimos se proporcionan al predictor cálculos automáticos a partir de la salida de un SPC cualquiera para distintos alcances de predicción y distintos tipos de aviso. Los resultados de estos cálculos, en forma de mapas y tablas, incluyen, además de la probabilidad de superación del umbral y proporción de área afectada, el nivel de aviso máximo y el aviso más plausible (ver Figura 35.2). Este último, se define como el nivel máximo de aviso que cumple simultáneamente dos condiciones: tener una probabilidad de superación del umbral superior al $40 \%$ y una proporción de área afectada de al menos el $20 \%$. Los resultados se presentan por comunidades autónomas, provincias, y zonas de aviso, tal como se definen en Meteoalerta.
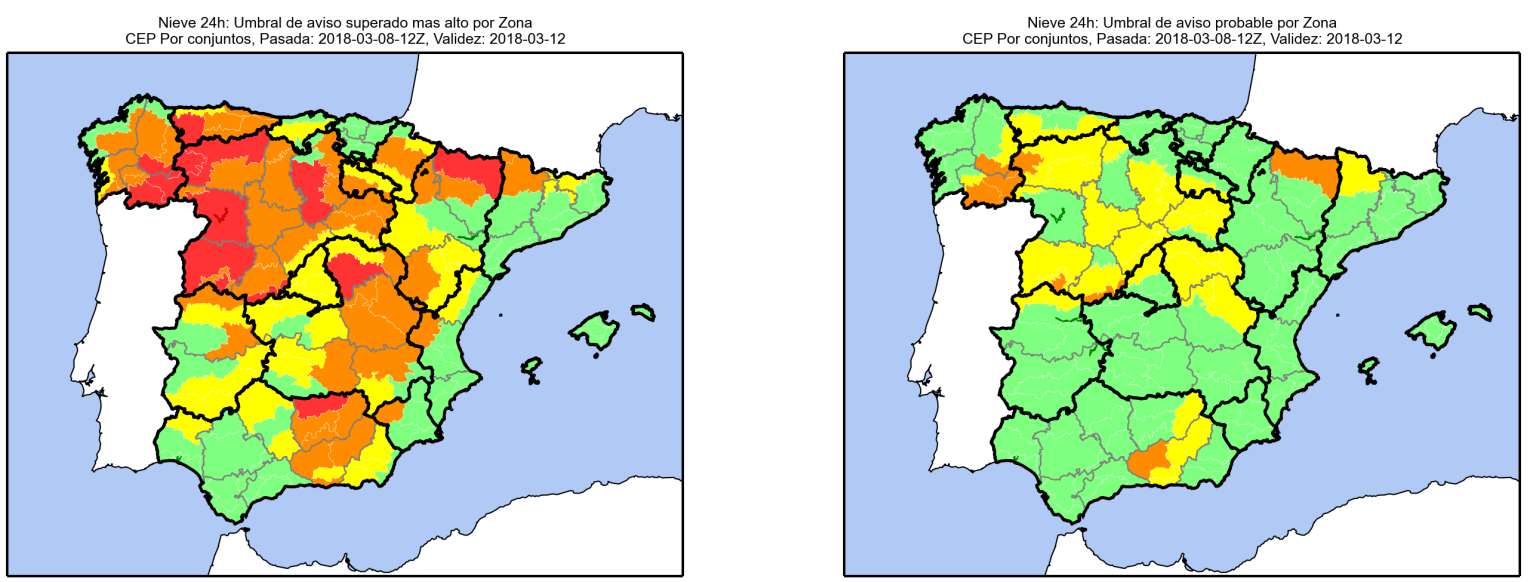

Figura 35.2: Nivel de aviso máximo por zona (izquierda) y niveles de aviso más plausibles, con probabilidad de superación de umbral de al menos $40 \%$ y una proporción de área afectada de al menos el $20 \%$ (derecha). 

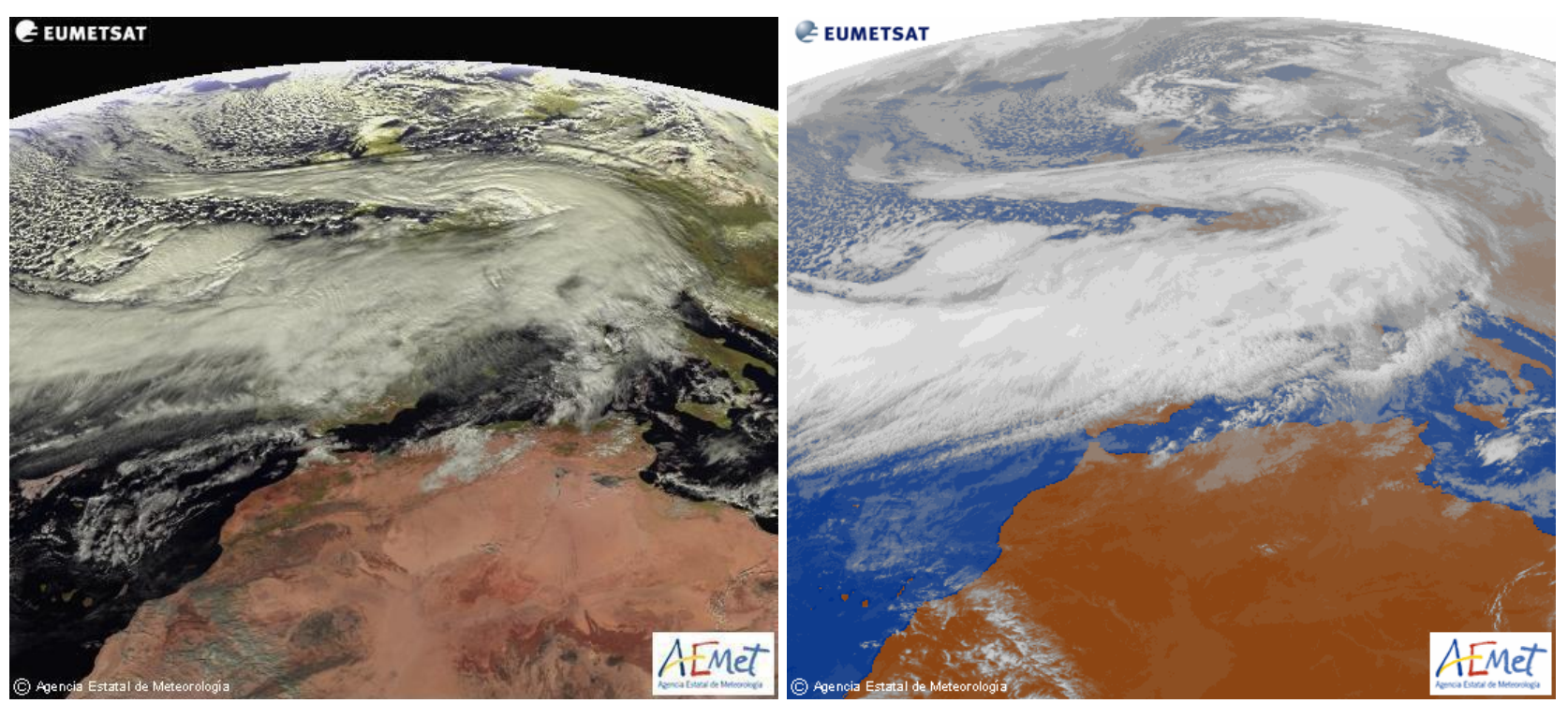

Figura 35.3: Imágenes del satélite MSG a las 12 UTC del 10-12-2017. A la izquierda, canal visible; a la derecha, canal infrarrojo (ambas imágenes están coloreadas).

Como decíamos, realizar un resumen de lo que proporciona un SPC tiene utilidad. Resumir es reducir la dimensión de la información inicial y un buen resumen es aquel que refleja los aspectos de la información inicial que nos interesan para una determinada aplicación. En este caso, al tratarse de avisos por fenómenos adversos, interesan los valores extremos, de manera que la atención la dirigimos, como hemos visto, hacia los valores más adversos predichos por los miembros del SPC, dejando de lado, por ejemplo, los valores medios. Pero hay que tener en cuenta que, indefectiblemente, al hacer un resumen siempre se pierde información. Sin embargo, la pérdida de información no es la principal limitación de una aplicación como esta. Al usar un SPC para la elaboración de avisos por fenómenos meteorológicos adversos, hay que tener en cuenta los aspectos que no están bien representados en las predicciones numéricas. Por ejemplo, la orografía que usa un SPC como el ECENS está suavizada respecto de la orografía real, ya que la resolución del SPC, actualmente de unos $18 \mathrm{~km}$ (sec. 19.3 en la página 293 y cap. A en la página 927), no permite reflejar gran cantidad de detalles, como valles profundos y estrechos, o cimas montañosas abruptas. Estos accidentes orográficos, no representados convenientemente en el modelo, tienen una influencia nada despreciable en el viento, temperatura o precipitación previstos. Además, en un modelo se hacen simplificaciones y aproximaciones que deterioran la calidad de las predicciones. Por tanto, hay que tener en cuen- ta las limitaciones del SPC de partida, para utilizar adecuadamente las predicciones que proporciona. $\mathrm{La}$ gran calidad de las predicciones finales, a pesar de las limitaciones que comentamos, ponen de manifiesto el importante valor del predictor humano como pieza esencial en los sistemas predictivos en la actualidad.

\subsection{Caso de estudio: la borrasca Ana}

Describimos brevemente la situación de los días 10 y 11 de diciembre de 2017, durante los cuales una borrasca, bautizada como ANA (las borrascas empezaron a bautizarse en España a finales de 2017), afectó principalmente a zonas del noroeste peninsular.

El centro de la borrasca se situaba al sureste de las islas británicas, con abundante nubosidad asociada a un frente muy activo (Figura 35.3). En niveles altos, $300 \mathrm{hPa}$, se formó rápidamente una vaguada con chorros asociados a ambos lados (Figura 35.4 en la página siguiente). En niveles medios, $500 \mathrm{hPa}$, había una masa fría aislada en el centro de la vaguada, con temperaturas mínimas de $-36{ }^{\circ} \mathrm{C}$. A las 00 UTC del día 11, el mínimo de presión estaba cercano a $965 \mathrm{hPa}$, al norte del mar Cantábrico. Este centro de bajas presiones tenía asociada una amplia zona con un fuerte gradiente de presión en superficie con vientos fuertes (ver Figura 35.4 en la página siguiente). 

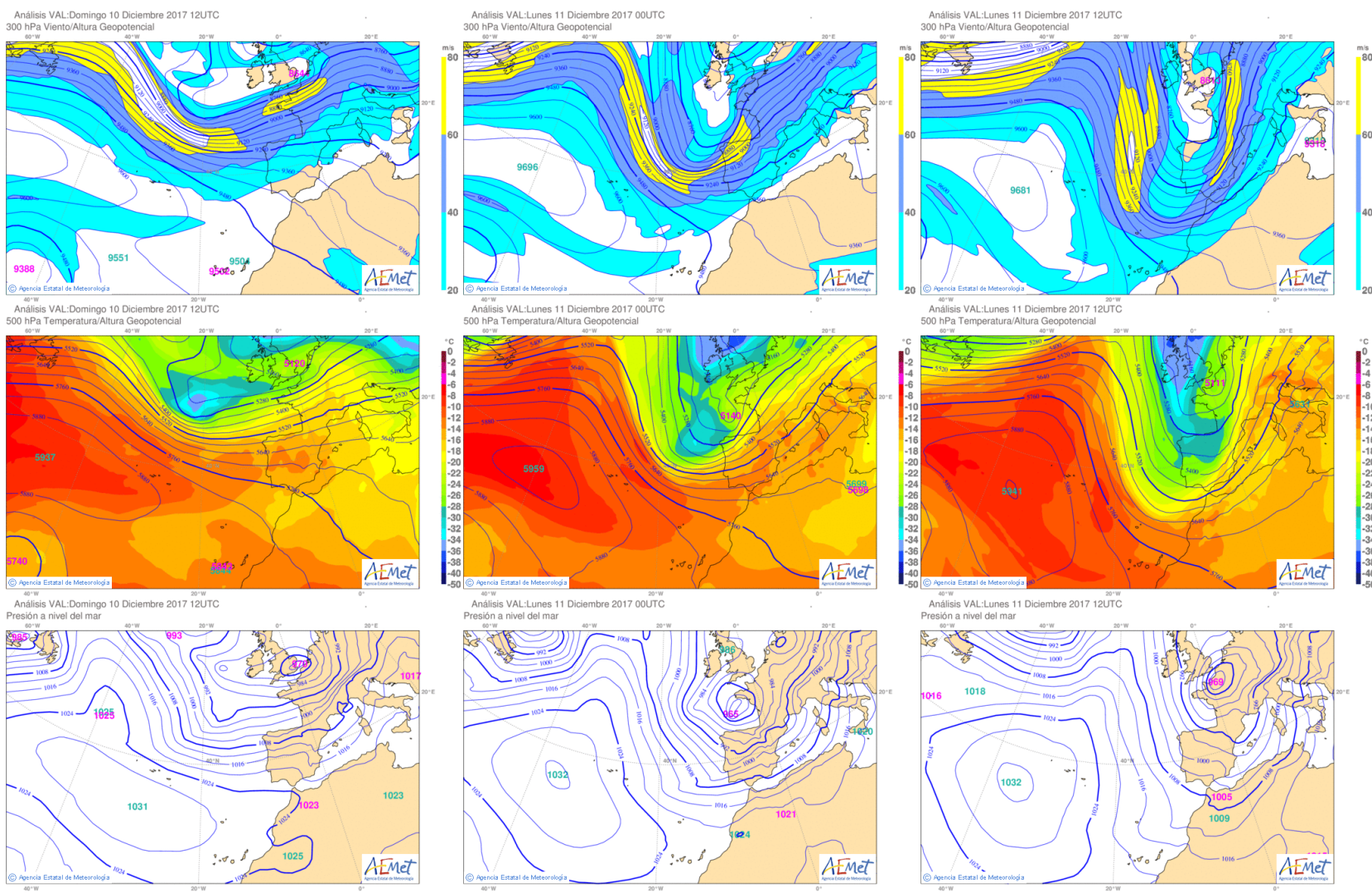

Figura 35.4: Evolución de la situación sinóptica, en columnas 10-12-2017 a las 12 UTC, 11-12-2017 a las 00 UTC y 11-12-2017 a las 12 UTC. Arriba, geopotencial y velocidad en $300 \mathrm{hPa}$; en medio, geopotencial y temperatura en 500 hPa; abajo, presión reducida a nivel del mar.

Se emitió un aviso especial de fenómenos adversos. AEMET predijo vientos muy fuertes, muy mal estado de la mar y lluvias intensas y persistentes en zonas del norte peninsular. En el noroeste y zonas montañosas el aviso mencionaba acumulaciones máximas entre 80 y $100 \mathrm{~mm}$. El fenómeno adverso más significativo fue el viento, fuerte y muy fuerte, del oeste y suroeste, en amplias zonas de la Península y Baleares, con rachas que podrían superar los $90 \mathrm{~km} / \mathrm{h}$ de forma generalizada, $110 \mathrm{~km} / \mathrm{h}$ en amplias zonas de la mitad norte, e incluso $130 \mathrm{~km} / \mathrm{h}$ en zonas montañosas de la mitad norte y Galicia.

Según la red de estaciones automáticas de AEMET, las rachas máximas de viento superaron los $140 \mathrm{~km} / \mathrm{h}$, llegando puntualmente a superar los $180 \mathrm{~km} / \mathrm{h}$ (Figura 35.6 en la página 577). Los $120 \mathrm{~km} / \mathrm{h}$ se superaron en zonas de Galicia, Castilla y León, Asturias, Santander y Euskadi principalmente. Puntualmente se superaron los $100 \mathrm{~km} / \mathrm{h}$ en áreas de la mitad sur Peninsular. Hay que tener en cuenta que el viento puede variar significativamente entre lugares relativamente cercanos y que, por tanto, se requiere una red densa de estaciones automáticas para reflejar de forma realista el campo de viento. La red de estaciones automáticas de AEMET puede ser insuficiente en algunas áreas.

Según las noticias publicadas, se produjeron diversos incidentes debido al viento: árboles caídos, problemas con el tendido eléctrico, cancelación de vuelos y de tráfico marítimo, así como cierre de algunas instalaciones como parques.

Consideramos ahora las predicciones del SPC del ECMWF, el ECENS, del día anterior, 9-12-2017, válidas para el día 10-12-2017. En cuanto a la probabilidad de superación del umbral (ver Figura 35.5 en la página siguiente, figuras en el lado izquierdo) las predicciones indicaban que era muy probable (probabilidad superior al 70\%) que se superara el umbral amarillo en amplias zonas del norte peninsular, e incluso en áreas del este peninsular. El nivel naranja se daba como muy probable en zonas de Galicia únicamente y, como probable (probabilidad entre $40 \mathrm{y}$ el $70 \%$ ), en Galicia y zonas de Asturias, norte de Castilla y León, Cantabria y Euskadi. Finalmente, en nivel de aviso rojo se preveía como posible en zonas de Galicia y como improbable en zonas del norte y este Peninsular. 

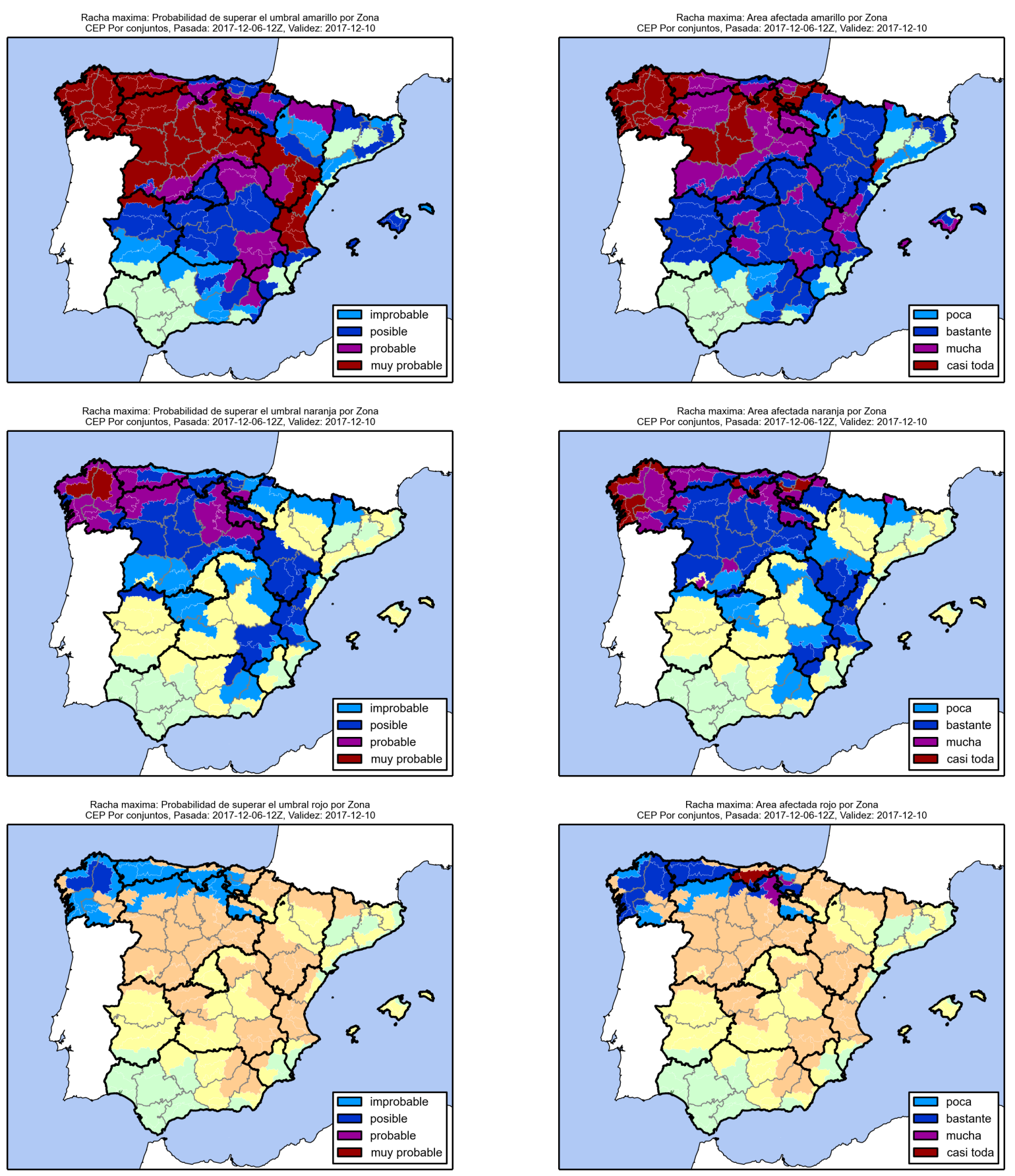

Figura 35.5: Avisos por rachas de viento: probabilidad de superación del umbral (izquierda) y proporción de área afectada (derecha) para el día 10-12-2017. Las filas corresponden a los umbrales de amarillo, naranja y rojo.

Se observa que el umbral rojo se superó en bastantes zonas que, en principio, se daban como poco probables o improbables (comparando las Figuras 35.5 y 35.6 en la página siguiente). La predicción parece que se quedó algo corta (no obstante, hay que tener en cuenta que no se puede verificar una predicción probabilista con un solo caso, ver cap. 15 en la página 207).

En cuanto a la proporción del área afectada (ver Figura 35.5, figuras en el lado derecho), la predicción indicaba la superación del umbral amarillo en casi todo el área (proporción superior al 70\%) en zonas de Galicia, Castilla y León y Euskadi. El nivel naranja también se preveía como fenómeno extenso (con proporciones superiores al $40 \%$ de área afectada) en prácticamente toda Galicia, zonas de Asturias, Cantabria, norte de Castilla y León y Euskadi. Finalmente, el nivel rojo se preveía menos extenso, con bastante área afectada (entre el 10 y el $40 \%$ ) en zonas de Galicia, norte de Castilla-León, Asturias, Cantabria y Euskadi. Pocas zonas presentaban proporciones de área afectada superiores al $70 \%$. 


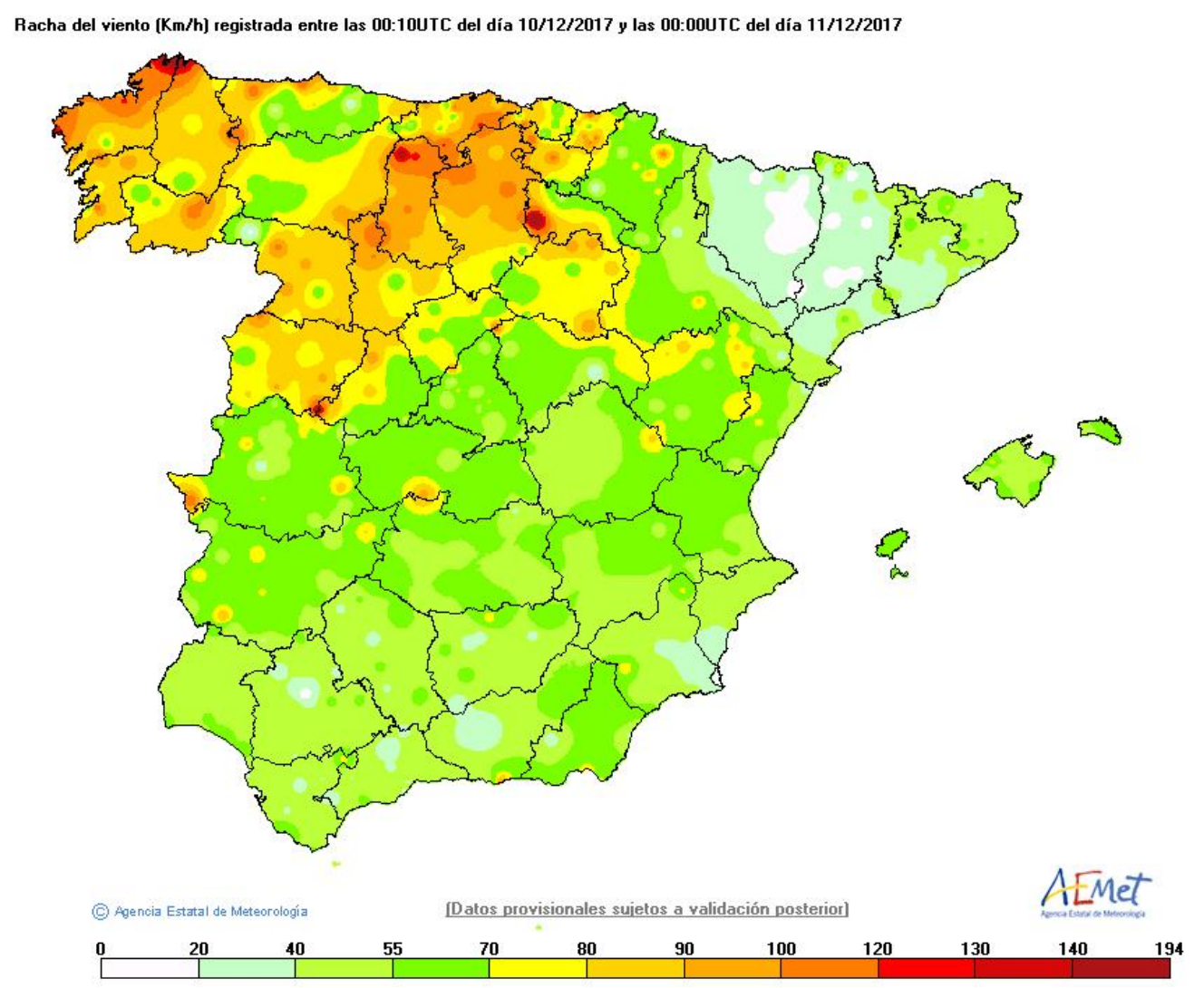

Figura 35.6: Rachas máximas de viento observadas durante todo el día 10-12-2017, según la red de estaciones automáticas de AEMET. 


\subsection{Referencias}

[1] AEMET. Plan Nacional de Predicción y Vigilancia de Fenómenos Meteorológicos Adversos. Informe técnico. Madrid: Agencia Estatal de Meterología (AEMET), 2015, página 56. URL: http : / / www . aemet . es / documentos / es / eltiempo/prediccion/avisos/plan_ meteoalerta/plan_meteoalerta.pdf (citado en página 570).

[2] Lalaurette, François. "Early detection of abnormal weather conditions using a probabilistic extreme forecast index". En: Quarterly Journal of the Royal Meteorological Society 129.594 (oct. de 2003), páginas 3037-3057. ISSN: 00359009. DOI: 10 . 1256/qj . 02.152 (citado en página 570).

[3] Molteni, F. y col. "The ECMWF ensemble prediction system: Methodology and validation". En: Quarterly Journal of the Royal Meteorological Society 122.529 (ene. de 1996), páginas 73-119. ISSN: 1477-870X. DOI: 10.1002 / qj . 49712252905 (citado en página 570 ). 
